\title{
Research of the synthesis of radiant intensity indicatrix of multi- component beam diode module
}

\author{
Ruslan Yakovliev ${ }^{1}$, Yurii Shmelov ${ }^{1, *}$, Maryna Petchenko ${ }^{1}$, Serhii Honchar ${ }^{1}$, and Vasyl Kovalskyi ${ }^{1}$ \\ ${ }^{1}$ Kharkiv National University of Internal Affairs, Kremenchuk flight college, 17/6 Peremogy Str., Kremenchuk, 39600, Ukraine
}

\begin{abstract}
Widespread use of semiconductor radiation sources in optoelectronic devices for various purposes requires further study of the mechanisms of formation of photometric characteristics of the integrated device in the near illumination zone, where the law of inverted squares is violated. A mathematical model of the multicomponent beam-diode module is proposed. On its basis the analysis of influence of the parameters of separate beam sources on the deformation of the indicatrix of radiant intensity at transition from the far zone to the near one is carried out. It is shown that the determining parameter of the indicatrix change in longitude and polar distance is the distribution in the plane of the modulus of the product of the radiant intensity of a single diode on its polar radius vector. The displacement of the polar angle of the maximum of the vector of the diode radiant intensity is more significant for wide radiation patterns than for concentrated ones. For specific parameters of diodes and geometry of their location the suitability of the proposed model for a priori modeling of beam-diode modules is illustrated.
\end{abstract}

\section{Introduction}

Successful development of unmanned aerial vehicles encourages the development and improvement of optoelectronic devices (OED) for light signals and reconnaissance purposes. They are mainly implemented by introduction of new element base of higher quality where not the last place belongs to artificial sources of infrared (IR) radiation. Increasing the range of security and reconnaissance OEDs is achieved by their transition to active mode, increasing the power of sources, the use of longer wavelengths of the IR range of electromagnetic waves (EMW). Among the artificial sources of spontaneous radiation, undoubtedly, the most promising are the semiconductor sources of injection electroluminescence of $\mathrm{p}$-n junctions - light-emitting diodes (LEDs) (visible area of EMW) and beam diodes (ultraviolet and infrared areas of EMW). Beam-signal and other devices of active action of the IR range have significant operational advantages at the working lengths of the EMW, which correspond to the "windows" of the transparency of the atmosphere in the middle range of the IR spectrum: $(3.3-4.2) \mu \mathrm{m},(4.5-5.1) \mu \mathrm{m},(8-13)$ $\mu \mathrm{m}$. Modern technological advances in the production of ultra-bright p-n-emitters in these parts of the spectrum require further study of the mechanisms of formation of the basic physical and technical characteristics of multicomponent beam-diode sources of directional action.

Due to the topicality of replacing traditional light sources with more efficient ones in many respects, a large number of scientific papers are devoted to the development and design of LED-based lighting devices.
Most of the papers are limited to offering a mathematical model for calculating the lighting characteristics of the LED module and computer software for these calculations. This is enough to perform practical tasks and the problem consists only in choosing the most rational model. From a theoretical point of view, it is important to study the peculiarities of the formation of light intensity curves (LIC) for the development of light intensity algebra. The non-triviality of these questions lies in the specific use of the category of light intensity for non-point sources. By definition, the intensity of light is a characteristic of a point source located at the apex of the solid angle and to arithmetically add vectors of light intensity from several sources is valid only for vectors of one direction when the source is at one point $[1,2,3]$. The method of virtual transfer of radiating components to one point to obtain a diagram of the direction of light intensity of the integrated device can be approximated only in the far illumination zone. The transition to the near zone raises the question of the legitimacy of the use of the light intensity characteristic.

Obviously, the pattern of light intensity or LIC of the integrated lighting device depends on the lighting characteristics of individual LEDs and the geometry of their placement. In principle, the LIC of any LED, even with secondary optics, can be calculated by considering the course of the beams in a semiconductor crystal $[4,5$, 6]. However, in practice a less costly approach is used. A simplified parametric model is selected for the empirically obtained data set. Such analytical models are the basis for the classification of the main types of LIC for sources of any radiation mechanism [7, 8, 9]. Together with the idealized diagram $\mathrm{f}(\theta)=\cos ^{-3} \cdot \theta(\theta-\mathrm{a}$

\footnotetext{
* Corresponding author: klk.nauka@gmail.com
} 
polar distance) there are 8 such types. Using the specified classification or point-discrete representation of empirical data for LIC diodes, the coordinate distribution of illumination from the multicomponent module $\mathrm{E}(\mathrm{x}, \mathrm{y})$ is determined as follows:

$$
E(x, y)=\sum_{i} \sum_{j}(I(x, y, i, j) \cdot \cos [\theta(x, y, i, j)]) /\left((x-i \Delta x)^{2}+\right.
$$

where $i$ - the number of LEDs along axis $\mathrm{x}$ with distance $\Delta x$ between them; $j$ - the number of LEDs along axis $y$ with distance $\Delta y$ between them; $I(x, y, i, j)$ - the intensity of light of the LED; $l$ - the distance from the LED module to the lighting plane; $\theta(x, y, i, j)$ - the angle of incidence of light from a single LED.

Due to the cumbersomeness of formulas and the specificity of simulation modeling, the identification of physical patterns of formation of integrated characteristics is complicated. Numerical results do not reflect the functional relationships of the characteristics of the isolated LED with the characteristics of the module. Therefore, the patterns of formation of integrated parameters of LED modules, determined at present, are the result of the analysis of graphical dependencies and are expressed verbally.

Basically, they are as follows:

1. The most uniform illumination can be provided by $[10,11,12]$ :

- modules of the cluster variant with a large number of low-power LEDs;

- placement of powerful LEDs in groups with nonconcentrated LIC;

- turns of LED, which is convenient in this case because of small occupied area and storage of a light flow.

2. When the module size is compared with the observation range, the illuminance with a change in distance changes insignificantly for non-turned diodes and for turned diodes with LIC with large radiation angles [14-18].

3. The anomalous coordinate dependence of the deviation coefficient on the law of inverse squares $K(r)$ is manifested when the beams are focused. Maximum values $K(r)$ increase as the angles of radiation of the LED decrease. With anomalous $K(r)$, value $E(r)$ sharply changes in the near illumination zone [13,14, 17-19].

4. Normal dependences of $K(r)$ are observed when using unturned LEDs with cosine-type LIC, as well as when defocusing the module [14, 16, 15, 19, 20-22].

5 . The more gently sloping dependences $K(r)$ are the less the decrease of $E(r)$ is [13, 18, 19, 23-24].

6. Dependences of relative uniformity of light distribution (RULD) are non-monotonic with minima in the near zone. For the near zone, defocusing results in higher RULD values than focusing [16-19].

7. High RULDs are achieved by reducing the maximum illuminance [16-22].

Purpose of the paper - to analytically research the peculiarities of the formation of the spatial distribution of the radiant intensity of the integrated beam-diode module, depending on the parameters of its components.

\section{Results}

For p-n-diode emitters with a spectrum outside the visible range the term "beam diode" is more correct than the term "LED". Suppose we have an integrated planar module assembled from $N$ beam-diode point sources. The irradiance at the observation point $A$ of the parallel plane (Fig. 1) is determined as follows:

$E(r, \theta, \varphi)=\sum_{k=1} I^{N}\left(I_{k}\left(\theta_{k}, \alpha_{k}, \rho_{k}, \varphi_{k}\right)\right) /\left(L_{k}^{2}\left(r, \theta, \varphi, \rho_{k}, \varphi_{k}\right)\right) \cdot \cos \theta_{k},(2)$

where $L_{k}\left(r, \theta, \varphi, \rho_{k}, \varphi_{k}\right)$ - the distance from the $k$-th source to the point of observation; $\theta_{k}$ - polar distance of spherical coordinates with the pole at the location of the $k$-th source; $r, \theta, \varphi$ - radius vector, polar distance, longitude of spherical coordinates with pole at selected point of module plane; $\rho_{k}, \varphi_{k}$ - the polar coordinates of the $k$-th source; $\alpha k$ - half the width of the radiation pattern of the $k$-th source; $I_{k}\left(\theta_{k}, \alpha_{k}, \rho_{k}, \varphi_{k}\right)-$ radiant intensity (light intensity) of the $k$-th source.

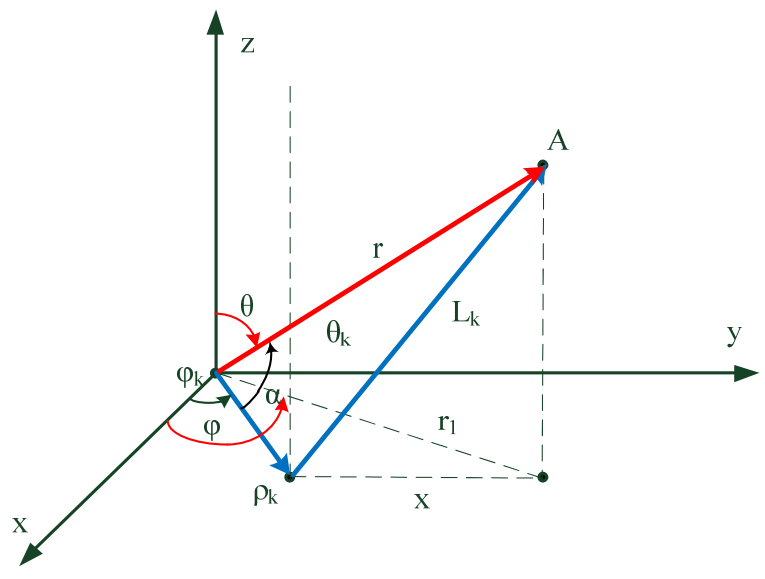

Fig. 1. The geometry of the analysis of illumination at point A from a point source with polar coordinates $\rho_{\mathrm{k}}, \varphi_{\mathrm{k}}$.

Taking into account that (Fig. 1):

$$
L_{k}\left(r, \theta, \varphi, \rho_{k}, \varphi_{k}\right)=r \cdot \sqrt{ }\left(1-2 r_{k} \cdot \sin \theta \cdot \cos \Delta \varphi_{k}+r_{k}^{2}\right)
$$

and

$$
\theta_{k}\left(r_{k}, \theta, \varphi\right)=\arccos \left(\cos \theta / \sqrt{ }\left(1-2 r_{k} \cdot \sin \theta \cdot \cos \Delta \varphi_{k}+r_{k}^{2}\right)\right),
$$

where $r_{k}=\rho_{k} / r ; \Delta \varphi_{k}=\varphi-\varphi_{k}$.

Expression (2) can be presented as follows:

$$
\begin{gathered}
E(r, \theta, \varphi)=\cos \theta / r^{2} \cdot \sum_{k=1}{ }^{N}\left(I_{k}\left(r, \theta, \Delta \varphi_{k}, \alpha_{k}, \rho_{k}\right)\right) / \\
{\left[1-2 r_{k} \cdot \sin \theta \cdot \cos \Delta \varphi_{k}+r_{k}^{2}\right]^{3 / 2} .}
\end{gathered}
$$

With $\quad r_{k} \rightarrow 0 \quad$ irradiation $\quad E\left(r, \theta, \varphi_{k}\right) \rightarrow \cos \theta / r_{2} \cdot \sum_{k=1} I_{k}$ $\left(\theta, \alpha_{k}\right)$, describing the irradiance of the module as a point source with radiant intensity $\sum_{k=1} I_{k}\left(\theta, \alpha_{k}\right)$. Based on this and the form of expression (3), the value

$$
\begin{gathered}
\left.I_{m}(r, \theta, \varphi)=\sum_{k=I^{N}}\left[I_{k}\left(r, \theta, \Delta \varphi_{k}, \alpha_{k}, \rho_{k}\right)\right]\right] \\
{\left[1-2 r_{k} \cdot \sin \theta \cdot \cos \Delta \varphi k+r_{k}^{2}\right]^{3 / 2},}
\end{gathered}
$$

where $r_{k}^{2}-2 r_{k} \cdot \sin \theta \cdot \cos \Delta \varphi_{k} \neq 1$ can be conditionally interpreted as radiant intensity of the module at $r \gg \rho_{k}$, i.e. 
for far illumination. In the near zone, where the coefficient of deviation of the irradiance from the law of inverse squares $K(r)<0.95$, such an interpretation of expression (4) is problematic, because at such distances from the module it is far from point one and for it the classical definition of radiant intensity cannot be applied.

Multiplier $\psi k(r, \theta, \varphi)=\left[1-2 r_{k} \cdot \sin \theta \cdot \cos \Delta \varphi_{k}+r_{k}^{2}\right]^{-3 / 2}$ takes into account the influence of a single source on the radiant intensity of the module depending on its location, or, on the other hand, takes into account its contribution to the "non-point nature" of the module. For the ease of analysis, we present $\psi_{k}$ as a power series. So, provided $\left|2 \mathrm{r}_{\mathrm{k}} \cdot \sin \theta \cdot \cos \Delta \varphi_{\mathrm{k}}-\mathrm{r}_{\mathrm{k}}{ }^{2}\right|<1$, which corresponds to the variation of $r$ from $r \gtrsim \rho_{k} / 2.4$ at $\sin \theta \cdot \cos \Delta \varphi_{k}=1$ to $r \gtrsim \rho_{k}$ at $\sin \theta \cdot \cos \Delta \varphi_{k}=0$, we have [10]:

$$
\begin{gathered}
\psi_{k}(r, \theta, \varphi)=1 / \Gamma(3 / 2) \cdot \sum_{n=0}{ }^{\infty} \Gamma(n+3 / 2) / n ! \cdot \\
\left(2 r_{k} \cdot \sin \theta \cdot \cos \Delta \varphi_{k}-r_{k}^{2}\right) n,
\end{gathered}
$$

where $\Gamma(x)=\int_{0}^{\infty} t^{\mathrm{x}-1} \cdot e^{-t} \cdot d t,(\operatorname{Re} x>0)-$ gamma function $[25$, 26].

Condition $r>\rho_{k}$ is sufficient for the legitimacy of the representation of $\psi_{k}$ by expression (5) with arbitrary values of $\theta$ and $\Delta \varphi_{k}$. Satisfied with the approximation at $\max n=1$, we have:

$$
\psi_{k}(r, \theta, \varphi) \cong 1+3 r_{k} \cdot \sin \theta \cdot \cos \Delta \varphi_{k}-3 / 2 r_{k}^{2}
$$

Then the radiation pattern of the modulus of radiation taking into account (4) and (6) can be represented in the following way:

$$
\begin{aligned}
f(r, \theta, \varphi) \cong & \sum_{\mathrm{k}=1}^{\mathrm{N}}\left(I k / I_{0}\right)+(3 \sin \theta / r) \sum_{\mathrm{k}=1^{\mathrm{N}}}\left[I k\left(I_{k} \cdot \rho_{k}\right) / I_{0}\right] \\
& \cos \Delta \varphi_{k}-3 /\left(2 r^{2}\right) \sum_{\mathrm{k}=1} \mathrm{~N}\left(I_{k} \rho_{k}^{2}\right) / I_{0},
\end{aligned}
$$

where $I_{0}=\lim _{\mathrm{T}}(\mathrm{r} \rightarrow \infty) \sum \mathrm{k}=1^{\mathrm{N}} I_{k}\left(r, \theta_{k}, \alpha k, \rho_{k}, \varphi_{k}\right)$.

(To simplify expression (7), the record of the functional dependence $\left.\mathrm{I}_{\mathrm{k}}\left(\mathrm{r}, \theta_{\mathrm{k}}, \alpha_{\mathrm{k}}, \rho_{\mathrm{k}}, \varphi_{\mathrm{k}}\right)\right)$ is omitted.

The first term in (7) describes the pattern of the algebraic sum of the radiation intensity of all point sources that are components of the module virtually located in the spherical coordinate pole.

The second term characterizes the deformation of the diagram in the meridional and azimuthal planes and is determined by the weighted average radial size and azimuthal arrangement of sources ${ }^{-} r=\sum_{\mathrm{k}}\left(I_{k} \cdot \rho_{k} \cdot \cos \Delta \varphi_{k}\right) / I_{0}$.

The third term describes the effect on the axial value of the diagram $\theta=0$ of the average radiation-weighted square of radial size ${ }^{-} r^{2}=\sum_{\mathrm{k}}\left(I_{k} \cdot \rho_{k}^{2}\right) / I_{0}$.

With $r \gg{ }^{-} r, \mathrm{r}^{2}$, corresponding to the far illumination zone, the pattern is determined by the first term (7). Experimental measurements of LIC or radiation patterns describe the dependence that corresponds to the first term (7) because the measurements are made in the far zone at the length of photometry where the radiant intensity does not depend on the distance from the source. It is obvious that dependences (4) and (7) can be used to interpret empirical data obtained in the near illumination zone with a large approximation.

The radiation pattern of the module is fixed relative to the selected pole of the spherical coordinates and the shape of the diagram in the near zone depends on this choice. It is most effective to choose the pole at the point of the plane of the module with the largest statistical contribution of the radiation of the diodes to the total radiant intensity or in the center of the weighted average radiation size of the module $[27,28]$.

The configuration of the radiation pattern of the isolated beam-diode is fixed relative to the pole located at the point of placement of the diode at a time when in the integrated device it is measured relative to the selected center of the spherical coordinates. However, in the integrated device, a large number of diodes are located outside the center of the spherical coordinates. Let us analyze the influence of the distance of the isolated diode from the center of registration of radiation force.

Based on a Lambert emitter with polar coordinates $\left(\rho_{k}, \varphi_{k}\right)$ and axial symmetry of its own diagram, the diagram of its radiation intensity in the module can be represented as follows:

$$
\begin{gathered}
f_{k}(r, \theta, \varphi)=\left[\cos \left(P_{k} \cdot \theta_{k}\right) \cdot R\left(\theta_{k}, \alpha_{k}\right)\right] / \\
{\left[1-2 r_{k} \cdot \sin \theta \cdot \cos \Delta \varphi_{k}+r_{k}^{2}\right]^{3 / 2},}
\end{gathered}
$$

where $P_{k}=\pi /\left(3 \alpha_{\mathrm{k}}\right)$ - the concentration parameter of the Lambert pattern [29];

$R\left(\theta_{k}, \alpha_{k}\right)=H\left(\theta_{k}\right)-H\left(\theta_{k^{-}}\left(3 \cdot \alpha_{k}\right) / 2\right)$ - limiting function for polar distance $\theta_{k}$;

$H(z)$ - asymmetric unit function.

Fig. $2(a, b)$ contains the change of the shape of the meridional section $\left(\Delta \varphi_{k}=\varphi-\varphi k=0\right)$ of the indicatrix of the radiation intensity of a single diode when the position of the diode shifts relative to the pole of the spherical coordinates of the module. With the diode distance $\rho_{\mathrm{k}}=0.2 \cdot r$ the maximum length of the radiation force vector $\theta_{\max }$ shifts by a certain value depending on ${ }_{\alpha \mathrm{k}}$ and makes $45^{\circ}$ for $\alpha_{k}=\pi / 3$ and $15^{\circ}$ for $\alpha_{k}=\pi / 12$. Displacement $\theta_{\max }$ in plane $\Delta \varphi_{k}=0$ may theoretically reach $90^{\circ}$, in other meridional planes with $\Delta \varphi_{k} \neq 0$ it will be less significant. Comparison of dependences of the coefficient of illumination deviation from the law of inverted squares (LIS) $K_{r}\left(r_{k}\right)$ and $\theta_{\max }\left(r_{k}\right)$ (Fig. 2 e) shows that at the boundary of the near and far zones of illumination $\left(K_{r} \cong 0.95\right)$ displacement $\theta_{\max }$ for the Lambert source is $\sim 30^{\circ}$.

The deformation of the indicatrix in the azimuthal plane occurs in the direction of displacement of the diode relative to the pole and is more significant for diodes with smaller $\alpha_{k}$.

For the axial force of the beam, taking into account (8), we obtain:

$$
I_{\text {M.o }}(r)=\sum_{\mathrm{k}=1}{ }^{\mathrm{N}}\left\{\mathrm{I}_{0 \mathrm{k}} \cdot \cos \left[\mathrm{P}_{\mathrm{k}} \cdot \arccos 1 / \sqrt{ }\left(1+\mathrm{r}_{\mathrm{k}}^{2}\right)\right] \cdot \mathrm{R}\left(\mathrm{r}, \alpha_{\mathrm{k}}\right)\right\} /
$$

where $\mathrm{R}\left(\mathrm{r}_{\mathrm{k}}, \alpha_{\mathrm{k}}\right)=\mathrm{H}\left(\mathrm{r}_{\mathrm{k}}\right) \cdot \mathrm{H}\left(\mathrm{r}_{\mathrm{k}}-\operatorname{tg} 3 / 2 \alpha_{\mathrm{k}}\right)$;

$$
\mathrm{I}_{0 \mathrm{k}}=\mathrm{I}_{\mathrm{k}}\left(\theta_{\mathrm{k}}=0\right) \text {. }
$$

For the Lambert emitters $P_{k}=1$ axial radiant intensity $I_{\text {м.о.л }}$ of the module with the Lambert components takes the form:

$$
I_{\text {M.о.л }}(r)=\sum_{\mathrm{k}=1}{ }^{\mathrm{N}} \mathrm{I}_{0 \mathrm{k}} /\left(1+\mathrm{r}_{\mathrm{k}}^{2}\right)^{2}
$$




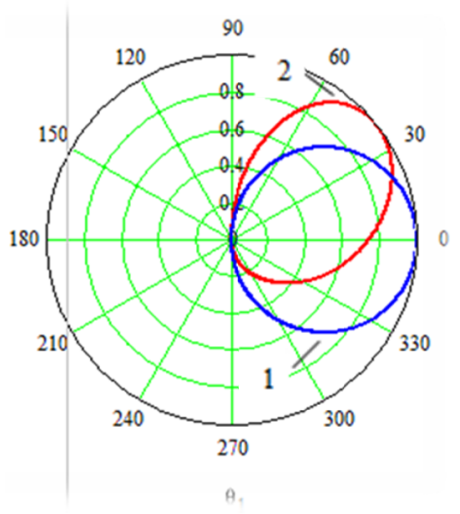

a

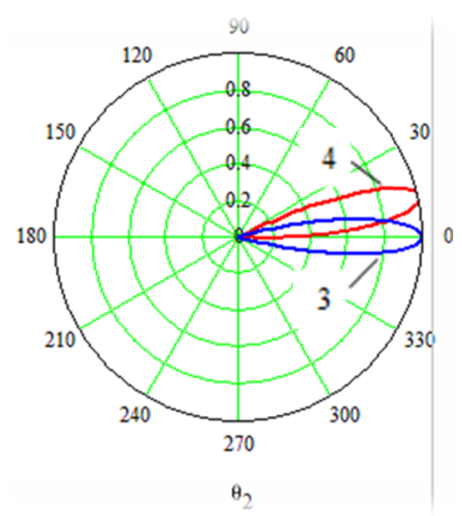

$\mathrm{b}$

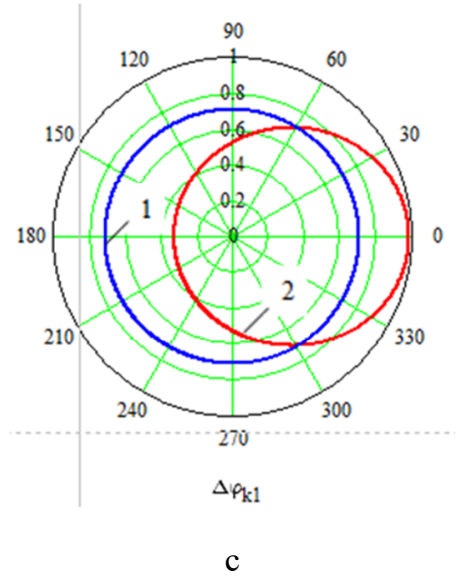

From expression (10) it follows: if $\mathrm{N}$ identical beamdiodes are placed radially symmetrically with respect to the pole (geometric center of the circle) then the total axial radiation intensity of the module $I^{*}{ }_{\text {м.олл }}(r)$ is:

$$
I_{\text {м.олл }}^{*}(r)=N \cdot I_{0 k} /\left(1+r_{k}^{2}\right)^{2}
$$

Fig. $4(a, b)$ contains the radiation patterns of the radiation of the module according to formula:

$$
\begin{array}{r}
\mathrm{f}(\mathrm{r}, \theta, \varphi)=1 / \mathrm{I}_{0} \sum_{\mathrm{k}=1}{ }^{\mathrm{N}}\left\{\mathrm{I}_{0 \mathrm{k}} \cos \left[\mathrm{P}_{\mathrm{k}} \cdot \theta_{\mathrm{k}}\left(\mathrm{r}_{\mathrm{k}}, \theta, \varphi\right)\right] \cdot \mathrm{R}\left[\theta_{\mathrm{k}}(\mathrm{r}, \theta, \varphi), \alpha_{\mathrm{k}}\right]\right\} / \\
\left(1-2 \mathrm{r}_{\mathrm{k}} \cdot \sin \theta \cdot \cos \Delta \varphi_{\mathrm{k}}+\mathrm{r}_{\mathrm{k}}^{2}\right)^{3 / 2}
\end{array}
$$

It should be noted that the radiation pattern for the axial radiant intensity expresses the coefficient of

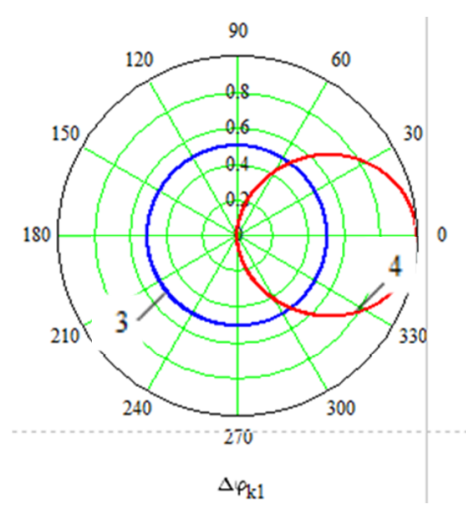

d

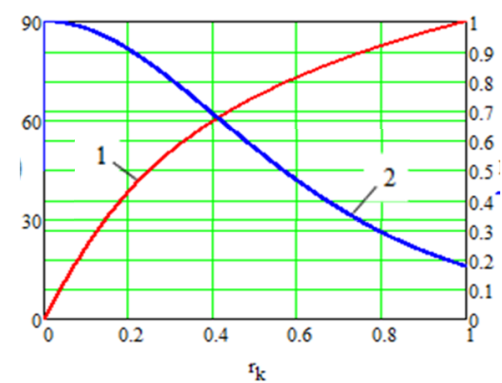

e

Fig. 2. Deformation of radiation patterns of diodes with $\alpha_{k}=\pi 3$ $(\mathrm{a}, \mathrm{c})$ and $\alpha_{k}=\pi / 12(\mathrm{~b}, \mathrm{~d})$ on frontal plane $\Delta \varphi_{\mathrm{k}}=0(\mathrm{a}, \mathrm{b})$ and azimuthal plane (c, d), curves: $1,3-r_{k}=0 ; 2,4-r_{k}=0.2 ; \mathrm{e}$ ) dependence $\theta_{\max }(1)$ and coefficient LIS (2) for the Lambert diode on $r_{k}=\rho_{k} / r$.

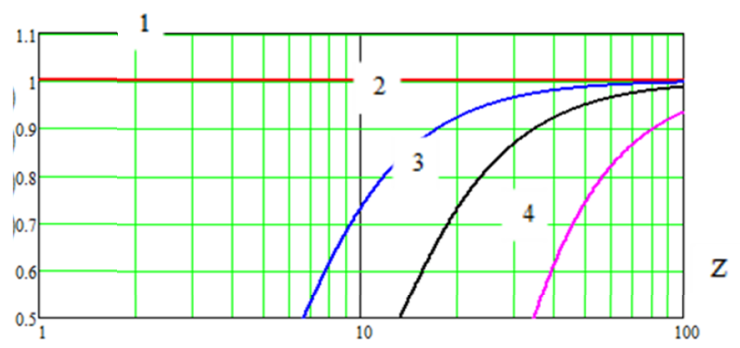

Fig. 3. Dependence of the normalized axial force of the diode radiation on the distance to the observation point $\mathrm{Z}$, curves: $1-r_{k}=0, \alpha_{k}=\pi / 6 ; 2-r_{k}=0.1, \alpha_{k}=\pi / 6 ; 3-r_{k}=0.2, \alpha_{k}=\pi / 6$; $4-r_{k}=0.1, \alpha_{k}=\pi / 36$.

deviation of irradiation (illuminance) from the law of inverse squares $K(r)$ :

$$
f(r, \theta=0)=K(r)=\left[J_{M}(r, \theta=0)\right] / I_{0} .
$$

\section{Conclusions}

1. The proposed analytical model of a multicomponent beam module is suitable for a priori modeling of light intensity curves (radiant intensity curves).

2. The proposed model includes:

a) the synthesis of radiant intensity and its indicatrix of the module integrated from point sources is performed according to the formulas (4), (12);

b) in the far illumination zone $\left(K_{k}>0.95\right)$ the indicatrix of the radiant intensity of the module does no 3

\footnotetext{
* Corresponding author: klk.nauka@gmail.com
} 
depend on the nature of the placement of the diodes and is determined by the first term of expression (7);

c) the deformation of the indicatrix of the radiant intensity of a multicomponent module during the transition from the far illumination zone to the near one depends on the geometry of the diodes relative to the pole of the spherical coordinates of the module and is determined by statistical values ${ }^{-} \mathrm{r}$ and ${ }^{-}\left(\mathrm{r}^{2}\right)$.

d) the displacement of the longitude of the maximum of the radiant intensity vector of a single diode depending on the distance to the coordinate pole is more significant for beam diodes with a wide pattern than with a concentrated one. The nature of the displacement of the azimuthal projection of the maximum of the vector is exactly the opposite.

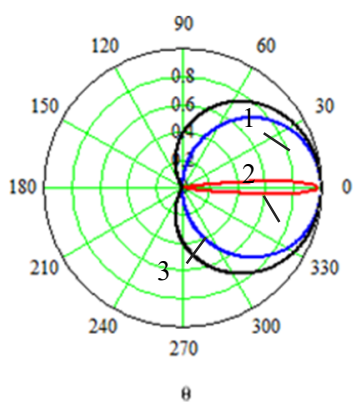

a

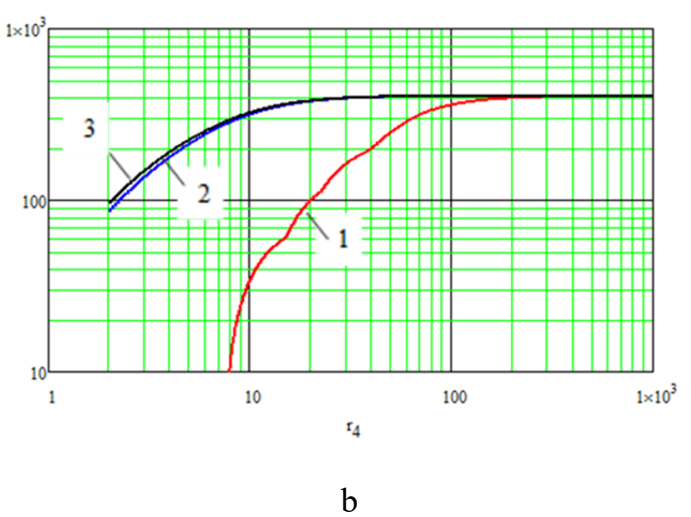

Fig. 4. a) radiation patterns of the radiant intensity in the frontal plane $(\varphi=0)$ of the module with diode parameters (13) for $\alpha_{k}$ : curve $1-\alpha_{k}=\pi / 36$; curve $2-\alpha_{k}=\pi / 3$;

curve $\left.3-\alpha_{k}=4 / 9 \pi ; b\right)$ dependence of the axial intensity of the radiation of the module on the distance to the observation point for diodes with different parameters: curve $1-\alpha_{k}=\pi / 36$; curve $2-\alpha_{k}=\pi / 3$; curve $3-\alpha_{k}=4 / 9 \pi$.

\section{References}

1. P.M. Tikhodeev, Light measurements in lighting engineering (ONTI, Moscow, 1936)

2. J. Bast, S.M. Gorbatyuk, I.Yu. Kryukov, Horizontal hec-12000 unit for the continuous casting of semifinished products. Metallurgist 55(1-2), 116118 (2011). doi:10.1007/s11015-011-9399-1

3. M.G. Naumova, I.G. Morozova, P.V. Borisov, Study of metal surface with color image obtained with laser marking. Solid State Phenomena 299
SSP, 943-948 (2020). doi:10.4028/www.scientific.net/SSP.299.943

4. A. Danilov, D. Borzov, Energy Performance Analysis of LEDs for Fiber Optic Converters. Actual problems of radio electronics 9, 40-44 (2004)

5. A. Shapoval et al Profitability of production of stainless steel + zirconium metals combination adapters. Key Engineering Materials 864, 285-291 (2020). doi:10.4028/www.scientific.net/KEM.864.285

6. O.A. Kobelev, S.V. Albul, N.L. Kirillova, Research and development of broaching methods on mandrel of large-sized pipe forgings. IOP Conference Series: Materials Science and Engineering 709(4), 044104 (2020). doi:10.1088/1757-899X/709/4/044104

7. I.V. Khovrak, M.V. Petchenko, Estimating the level of financial safety in banking institutions. Actual Problems of Economics 164 (2), 347-354 (2015)

8. O.E. Markov et al, Improvement of Upsetting Process of Four-Beam Workpieces Based on Computerized and Physical Modeling. FME Transactions 48(4), 946-953 (2020). doi: $10.5937 /$ fme2004946M

9. N. Hrudkina et al, Predicting the shape formation of hollow parts with a flange in the process of combined radial-reverse extrusion. EasternEuropean Journal of Enterprise Technologies 4 (1106), 55-62 (2020). doi:10.15587/17294061.2020.203988

10. T. Antonova, E. Gutzait, L. Kochan, A. Krasnopolskii, D. Miliutin, Calculations of illumination of workplaces by LEDs, in Reports of the 60th scientific session dedicated to Radio Day. Optoelectronics and fiber optic devices, pp. 39-41 (2005)

11. A.Yu. Zarapin, A.I. Shur, N.A. Chichenev, Improvement of the unit for rolling aluminum strip clad with corrosion-resistant steel. Steel in Translation 29(10), 69-71 (1999)

12. A. Keropyan, S. Albul, A. Zarapin, Problem of Increasing Tractive Effort of Railway Locomotives in Conditions of Arctic and Continental Shelf Regions. Lecture Notes in Mechanical Engineering, pp. 651-658 (2020). doi:10.1007/978-3-030-220631_69

13. E.M. Gutzait, Investigation of normal and anomalous characteristics of the coefficients of deviation from the law of the inverse square of the distance when using LED modules. Radio Engineering and Electronics 54(12), 1495-1512 (2009)

14. E.M. Gutzait, Investigation of illumination by LED modules located at big distances from the lighted objects. Radio Engineering and Electronics 1, 113124 (2009)

15. V. Artiukh, V. Mazur, A. Adamtsevich, Priority influence of horizontal forces at rolling on operation of main sheet rolling equipment. MATEC Web of 
Conferences $\quad$ 106, 04001

doi:10.1051/matecconf/201710604001

(2017).

16. Y. Shmelov, S. Vladov, Y. Klimova, M. Kirukhina, Expert system for identification of the technical state of the aircraft engine TV3-117 in flight modes, in 1st IEEE International Conference on System Analysis and Intelligent Computing, SAIC 2018, 8516864. doi:10.1109/SAIC.2018.8516864

17. A. Shapoval et al, Technology of Production of Refractory Composites for Plasma Technologies, in Proceedings of the 25th IEEE International Conference on Problems of Automated Electric Drive. Theory and Practice PAEP 2020, 9240830. doi:10.1109/PAEP49887.2020.9240830

18. E.M. Gutzait, W.E. Maslov, T.A. Agafonova, Results of calculations of illuminances from LED modules and research of anomalous characteristics of coefficients of deviation from the law of a square of distance, in 7th Belarusian-Russian seminar Semiconductor lasers and systems based on them, pp. 137-140 (2009)

19. E.M. Gutzait, V.E. Maslov, Analysis of the distribution of illumination in the near area from two-dimensional models with rotated LEDs. Semiconductor lighting 3 (2012)

20. T. Shmelova, Y. Shmelov, S. Vladov, Concept of Building Intelligent Control Systems for Aircraft, Unmanned Aerial Vehicles and Aircraft Engines, in 6th International Conference on Methods and Systems of Navigation and Motion Control, MSNMC 2020, $9255509 \quad$ (2020). doi:10.1109/MSNMC50359.2020.9255509

21. I. Savchenko, A. Shapoval, A. Gurenko, Modeling Dynamic Parameters of Hard Alloys during Shock Wave Regeneration. IOP Conference Series: Materials Science and Engineering 969(1), 012079 (2020). doi:10.1088/1757-899X/969/1/012079

22. V.V. Kondratenko et al, Static analysis and strength calculation of drive shaft of large-scale cone crusher. E3S Web of Conferences 193, 01038 (2020). doi:10.1051/e3sconf/202019301038

23. O. E. Markov et al, Computerized simulation of shortened ingots with a controlled crystallization for manufacturing of high-quality forgings. International Journal of Advanced Manufacturing Technology 103(5-8), 3057-3065 (2019). doi:10.1007/s00170-019-03749-4

24. S.N. Larin, V.I. Platonov, K.N. Solomonov, Approach to assessment of microdamages accumulated during the constrained molding of shells made of the material subject to energy theory of creep and damage. Journal of Chemical Technology and Metallurgy 52 (4), 679-684 (2017)

25. I.V. Petrunenko, S.I. Belei, M.V. Petchenko, N.V. Kovalenko, O.A. Bodnar, N.G. Maslak, Organizational and financial principles for the development of euroregions. International Journal of Economics and Business Administration 8(3), 150160 (2020). doi:10.35808/ijeba/493
26. O. Markov et al, Development of the metal rheology model of high-temperature deformation for modeling by finite element method. EUREKA, Physics and Engineering 2019 (2), 52-60 (2019)

27. E.V. Sobolev, E.N. Poddenezhnykh, Modeling of lighting characteristics of LED modules (2011)

28. M. Zagirnyak, V. Zagirnyak, D. Moloshtan, A search for technologies implementing a high fighting efficiency of the multilayered elements of military equipment. Eastern-European Journal of Enterprise Technologies 6 (1-102), 33-40 (2019)

29. A.A. Titova, Experimental and numerical calculation of LED patterns. SWORLD 7(4), 10-16 (2012) 